\title{
Android Based GIS Terminal Design in Tobacco Distribution
}

\author{
Hao-Bo LI ${ }^{\mathrm{a}}$, Hao-Liang LI, Ming-Yuan YANG and Na WANG \\ Zhengzhou University, School of Information Engineering, 450001 Zhengzhou, China
}

\begin{abstract}
With the development of intelligent devices, Android smart device is widely used in daily life. Based on android smart device, this paper aims to implement a multifunctional GIS mobile terminal which integrates Internet communication, bar-code scanning, and LBS (Location Based Service) technologies. The GIS terminal, based on Android application designing, make it possible to optimize the management in tobacco distribution. At last, test results prove that the design of the application development can meet the expected functional requirements of the GIS system.
\end{abstract}

\section{Introduction}

With the development of intelligent devices, a variety of portable intellectual equipment has been applied to various industries, especially Android based intelligent terminals. Since the open source of Android system, it has been widely used and further developed. The lower cost and easy operation advantages of the Android device make it possible for integrating Android based device into the tobacco distribution GIS system.

Based on Android platform, GIS mobile terminal can extend many function modules, and provides unique functions integrated with real-time positioning, route navigation, network communication, and bar-code scanning [1]. All the functions, as is described above, depend on the wireless network technology, especially $3 \mathrm{G}$ or $4 \mathrm{G}$ network.

In conclusion, this paper designs a GIS terminal software on Android platform. The main content of this paper is summarized in the following three sections. The section two introduces mobile positioning, wireless network, bar-code recognition, and database technology. The section three presents the software application designing. In section four, it introduces the potential problem and challenge in the design.

\section{The key technology}

\subsection{Mobile positioning technology}

Mobile positioning technology uses satellite and wireless network techniques to calculate current position, by receiving signals of the satellite or base-station $[1,2,3]$. It's an indispensable part of the GIS system. Mobile GPS technology needs multiple satellites to get device location. Via at least four

\footnotetext{
${ }^{\mathrm{a}}$ Corresponding author: ielihaobo@163.com
} 
satellites signal, it can calculate the target's location information by a specific algorithm [4]. Similarly, wireless network positioning uses the destination between client and base-station, computing the target location.

Compared with the traditional GPS positioning technology, Baidu Map could provide a more precise location service. Using Baidu Map service, the terminal can combine GPS and GPRS to get location. It can not only improve the positioning accuracy, but also reduce the power waste of the device. Using the Baidu Map can design a comprehensive, real-time monitoring program for GIS system. As shown in Figure.1, the Baidu Map can provide multiple features for user.

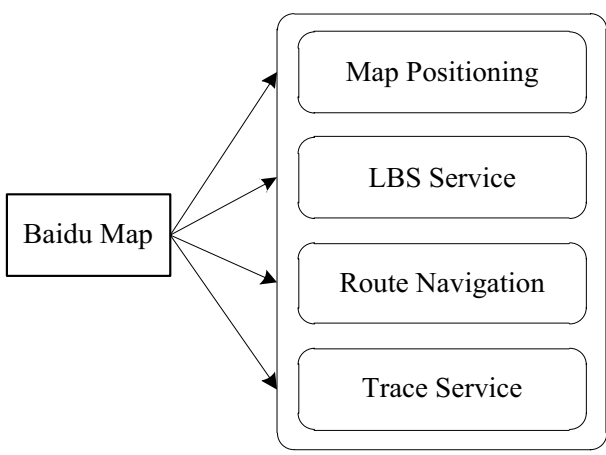

Figure 1. Some features of Baidu Map

\subsection{Wireless network technology}

Mobile terminal usually utilizes wireless network connect to the Internet [5]. The digital cellular network is the mainstream technology of mobile network.

Mature $3 \mathrm{G}$ and $4 \mathrm{G}$ network have been quite popular, especially $4 \mathrm{G}$ wireless network. With the development of network speed and signal quality, the wireless network technology can provide a very good environment for using of mobile GIS terminal.

\subsection{Image processing technology}

Camera equipment, providing photograph function, is usually integrated into Android devices. Native Android system contains camera software, which can open the camera to capture image or video information.

To start a camera hardware, software application calls camera interfaces in the Android system, and calls the camera driver to start the camera device, realizing image capture capability. Combined with image processing technology, the Android device enables to realize image recognition feature, identifying information from bar-codes or QR codes.

\subsection{Database technology}

Database technology uses a lightweight database to cache network data and geographical information data. Database cache technology will speed up data reload, and resolve the problem that data cannot be accessed from the Internet under the condition of network broken. Database designing in this paper will help improve work efficiency, facilitating data access for the user $[1,3]$.

In this design, the terminal software uses the database operation method add data to the database, after converting network data into a special data model. To read and upload data, the terminal will query data from database and package the data into special format, then using mobile network send data to the server. 


\section{Software specification of GIS}

\subsection{Basic map service}

Based on Android system, the GIS terminal can realize basic map service by using Baidu Map API. Baidu Map SDK offers rich functions such as map display and positioning, route planning, etc. Meanwhile, it provides query and playback method of historical data, that includes geographical information and customization data.

By combining GPS, GPRS, and Wi-Fi technology, the Baidu Map can obtain location information of the device. Based on this function, GIS terminal can get real-time location data of the device, then upload those data to the supervision and management system [5].

\subsection{Route navigation}

Traditional vehicle navigation system depends on the GPS technology. And the navigation software can analyze route data, providing route planning and guidance between current location and destination address.

Combined with basic road information and real-time traffic condition, Baidu Map navigation, depending on cloud computing, can provide users with various navigation plans under different constraints [6,7]. Using the Baidu Map navigation function, GIS terminal can recalculate navigation route under the condition of avoiding traffic congestion [8,9]. In the design of GIS software, a detection method is implemented to estimate whether the vehicle needs to recalculate navigation route. In Figure.2, it briefly shows the process of Baidu Map navigation.

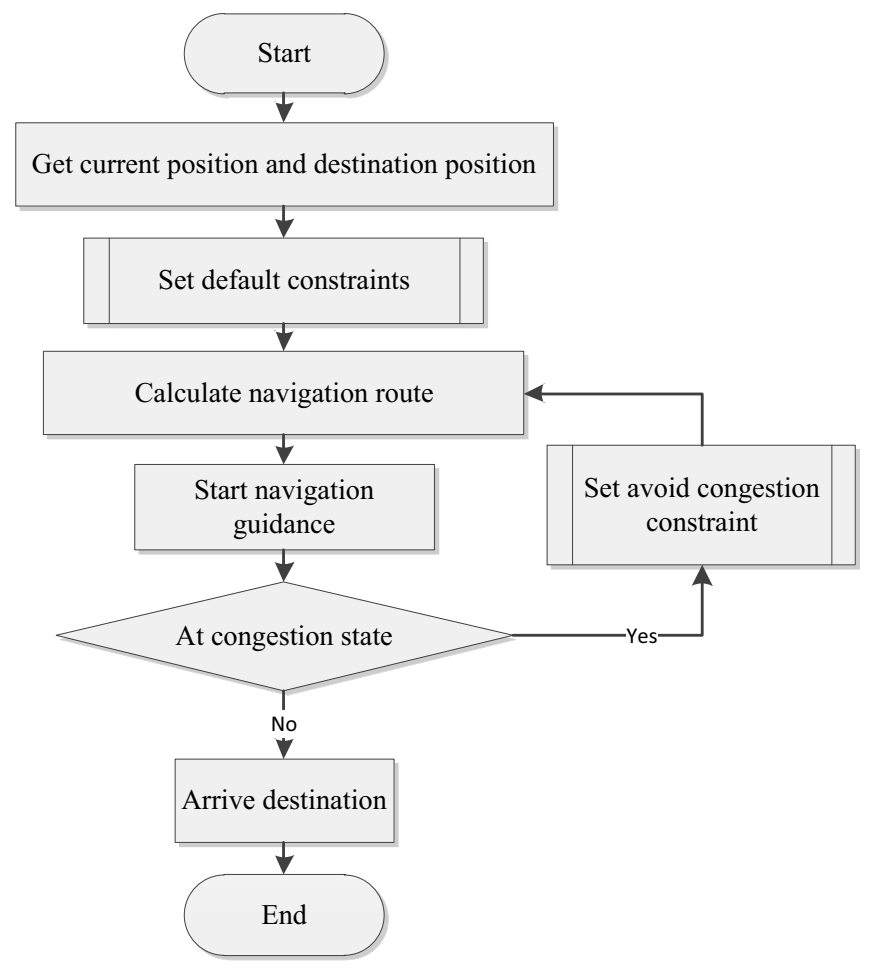

Figure 2. The process of Baidu Map Navigation 
In order to test the efficiency of the terminal, we made a comparison about the cost of the time and distance, delivering from Shop A to Shop B. In Table 1, we could see a clear cut down, compared with previous condition.

Table 1. The comparison of time and distance.

\begin{tabular}{|l|c|c|}
\hline & Time Cost & Traveling Distance \\
\hline Previous & $35 \mathrm{~min}$ & $13.3 \mathrm{~km}$ \\
\hline Now & $25 \mathrm{~min}$ & $12.8 \mathrm{~km}$ \\
\hline
\end{tabular}

\subsection{Wireless network communication}

Utilizing Android wireless network module and the Http network protocol, delivery personnel could transmit personal information to the supervision and management system [10,11]. Meanwhile, the terminal will start background service process to upload vehicle location information.

GIS terminal calls map positioning function to get current position information of the vehicle, and applies database operation method get local delivery state data. This information will be packaged into Json data format, and uploaded to the system server. To meet the requirement of real-time monitoring, GIS terminal device needs uninterrupted send data to the back-end server.

The terminal design uses the Http protocol to access the network data. The application software would package related information, and add working condition, geographical location and other information to the Http request. With wireless network data transmission technology, the terminal can transmit various information to the system, which will help back-end system get real-time distribution state and manage distribution process.

\subsection{Bar-code scanning}

Bar-code scanning technology is widely used in the logistics system to facilitate receipting of the order form. In this design, using the camera model, the terminal can parse digital information from bar-code image, that is taken by the terminal [12].

Utilizing digital image analysis technology, bar-code scanning device based on Android is different from traditional bar-code scanner. First, camera model of the device get the bar-code image. And then scanner passes over the image to produce a special stream. Through several steps decoding the stream, it will produce a stream of completely decoded symbol data. The decoded data result is extracted from the image. And finally, the result is passed to the user interface display. According to the result data, terminal can query database to get detail information of order form. The Figure. 3 shows the process of bar-code decoding.

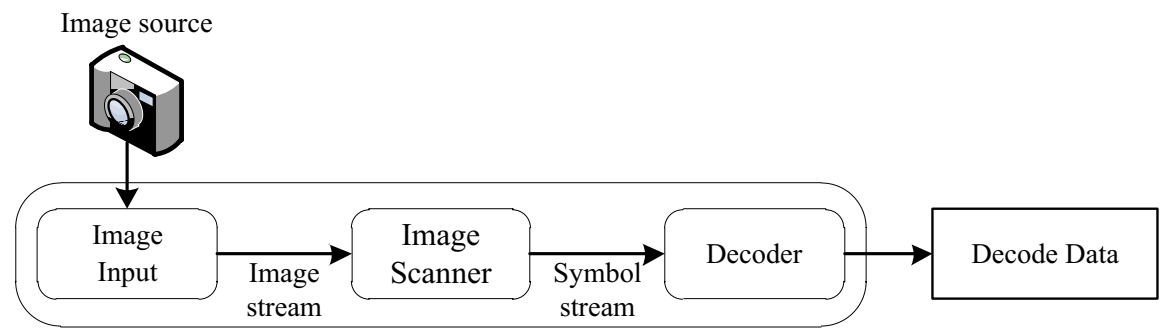

Figure 3. The process of bar-code decoding

When signing for the order, it usually spends about half an hour to completely confirm the order from. By using the function of bar-code scanning, it can be more easier and faster to finish this process. In Table 2, it shows a decrease of average time cost, during checking an order. 
Table 2. An average decrease of time cost.

\begin{tabular}{|l|c|c|}
\hline & Check Order & Sign for Order From \\
\hline Previous & $10 \mathrm{~min}$ & $5 \mathrm{~min}$ \\
\hline Now & $5 \mathrm{~min}$ & $2 \mathrm{~min}$ \\
\hline
\end{tabular}

\section{The challenge for GIS technology on android device}

GIS terminal applies wireless network to access to the back-end system, which will bring some network security risks. During data transmission, vehicle location and customer information could be leaked, that may cause huge economic losses to the operators and customers. In addition, the GIS terminals need uninterruptedly access to the network, leading to power consumption increase of the device. In summary, it requires application designers to optimize program design to overcome these challenges.

\section{Conclusion}

The conclusion of this paper can be summarized as following points. Using Baidu Map development tools, the terminal can provide positioning, route navigation and real-time guidance of route planning. Based on wireless network technology, this design implements real-time information interacting with the back-end system. And a local database is designed to cache the network data. It also integrates bar-code scanning technique to get order number from images. After completed and tested the terminal functions, the results show that the Android based GIS terminal is able to meet the expected requirements of GIS system.

\section{References}

1. Jingsong. Ge, Fang. Wang and Hui. Deng, 2013 6th International Conference on Intelligent Networks and Intelligent Systems (ICINIS), "An Investigation on GIS Technology for Android Embedded System", pp.312-315(2013).

2. J. Huang, Y. Zhan, W. Cui, Y. Yuan and P. Qi, 2010 International Conference on Computer Design and Applications, "Development of a campus information navigation system based on GIS", pp.491- 494(2010).

3. S. Exauty, H. Gao, X. Wang and X. Yu, 2016 International Conference on Logistics, Informatics and Service Sciences (LISS), "Design of navigation system and transmitter based on GPS and GSM", pp.1-4(2016).

4. H. Guan, L. Li and X. Jia, 2013 IEEE Third International Conference on Information Science and Technology (ICIST), "Multi-sensor fusion vehicle positioning based on Kalman Filter", pp.296-299(2013).

5. E. D'Andrea and F. Marcelloni, 2016 IEEE International Conference on Smart Computing (SMARTCOMP), "Incident Detection by Spatiotemporal Analysis of GPS Data", pp.1-5(2016).

6. P. Jeefoo, 2014 International Conference on Information Science \& Applications (ICISA), "Real-Time Field Survey Using Android-Based Interface of Mobile GIS", pp.1-3 (2014).

7. E. K. Hashi, M. R. Hasan and M. S. U. Zaman, 2016 19th International Conference on Computer and Information Technology (ICCIT), "GIS based heuristic solution of the vehicle routing problem to optimize the school bus routing and scheduling", pp.56-60(2016).

8. K. Yamamoto and T. Ikeda, 2016 IEEE 18th International Conference on High Performance Computing and Communications; IEEE 14th International Conference on Smart City; IEEE 2nd International Conference on Data Science and Systems (HPCC/SmartCity/DSS), "Social Recommendation GIS for Urban Tourist Spots", pp.50-57(2016). 
9. M. Fu, W. Song, Y. Yi and M. Wang, 2015 IEEE 18th International Conference on Intelligent Transportation Systems, "Path Planning and Decision Making for Autonomous Vehicle in Urban Environment", pp.686-692(2015).

10. A. Hrazdira, A. Cela, etc, 2012 15th International IEEE Conference on Intelligent Transportation Systems, "Optimal Real-Time Navigation System: Application to a Hybrid Electrical Vehicle", pp.409-414(2012).

11. P. K. Arora, R. Bhatia, S. Parkash and B. J. S. Sekhon, 2015 4th International Conference on Reliability, Infocom Technologies and Optimization (ICRITO) (Trends and Future Directions), "Web based GPS and GIS model for rural areas", pp.1-5(2015).

12. S. F. A. Razak, C. L. Liew, C. P. Lee and K. M. Lim, 2015 IEEE Student Conference on Research and Development, "Interactive android-based indoor parking lot vehicle locator using QR-code", pp.261-265(2015). 